\title{
Visual lung damage CT score at hospital admission of COVID-19 patients and 30-day mortality
}

\author{
Etienne Charpentier ${ }^{1,2} \cdot$ Gilles Soulat $^{1,2,3} \cdot$ Antoine Fayol $^{1,3,4} \cdot$ Anne Hernigou ${ }^{1,2} \cdot$ Marine Livrozet $^{1,4}$. \\ Teodor Grand $^{1,2}$. Guillaume Reverdito ${ }^{1,2}$. Jad al Haddad ${ }^{1,2} \cdot$ Kim Diep Dang Tran ${ }^{1,2}$. Anne Charpentier ${ }^{1,2}$. \\ Olivier Clement $^{1,2,3} \cdot$ Jean-Sebastien Hulot ${ }^{1,3,4} \cdot$ Elie Mousseaux ${ }^{1,2,3,5}$ (D)
}

Received: 24 October 2020 / Revised: 2 March 2021 / Accepted: 24 March 2021 / Published online: 29 April 2021

(C) European Society of Radiology 2021

\begin{abstract}
Objectives Chest CT has been widely used to screen and to evaluate the severity of COVID-19 disease in the early stages of infection without severe acute respiratory syndrome, but no prospective data are available to study the relationship between extent of lung damage and short-term mortality. The objective was to evaluate association between standardized simple visual lung damage CT score (vldCTs) at admission, which does not require any software, and 30-day mortality.

Methods In a single-center prospective cohort of COVID-19 patients included during 4 weeks, the presence and extent of ground glass opacities(GGO), consolidation opacities, or both of them were visually assessed in each of the 5 lung lobes (score from 0 to 4 per lobe depending on the percentage and out of 20 per patient $=$ vldCTs $)$ after the first chest $\mathrm{CT}$ performed to detect COVID-19 pneumonia.

Results Among 210 confirmed COVID-19 patients, the number of survivors and non-survivors was 162 (77\%) and 48 (23\%), respectively at 30 days. vldCTs was significantly higher in non-survivors, and the AUC of vldCTs to distinguish survivors and non-survivors was 0.72 (95\%CI $0.628-0.807, p<0.001)$; the best cut-off vldCTs value was 7 . During follow-up, significant differences in discharges and 30-day mortality were observed between patients with vldCTs $\geq 7$ versus vldCTs $<7:(98$ [85.2\%] vs 49 [51.6\%]; $p<0.001$ and 36 [37.9\%] vs $12[12.4 \%] ; p<0.001$, respectively. The 30-day mortality increased if vldCTs $\geq 7$ (HR, $3.16(1.50-6.43) ; p=0.001)$, independent of age, respiratory rate and oxygen saturation levels, and comorbidities at admission.

Conclusions By using chest CT in COVID-19 patients, extensive lung damage can be visually assessed with a score related to 30 day mortality independent of conventional risk factors of the disease.

Key Points

- In non-selected COVID-19 patients included prospectively during 4 weeks, the extent of ground glass opacities(GGO) and consolidation opacities evaluated by a simple visual score was related to 30-day mortality independent of age, respiratory rate, oxygen saturation levels, comorbidities, and hs-troponin I level at admission.

- This severity score should be incorporated into risk stratification algorithms and in structured chest CT reports requiring a standardized reading by radiologists in case of COVID-19.
\end{abstract}

Keywords COVID-19 $\cdot$ Pneumonia $\cdot$ Severe acute respiratory syndrome $\cdot$ Tomography, x-ray computed

Elie Mousseaux

elie.mousseaux@aphp.fr

Université de Paris, F-75015 Paris, France

2 Department of Radiology, Assistance Publique Hôpitaux des Hôpitaux de Paris (AP-HP), Hôpital Européen Georges-Pompidou (HEGP), Paris, France
3 Institut National de la Santé et de la Recherche Médicale, PARCC, UMR970, F-75015 Paris, France

4 CIC1418 and DMU CARTE, Assistance Publique Hôpitaux de Paris (AP-HP), Hôpital Européen Georges-Pompidou,

F-75015 Paris, France

5 Service de Radiologie, Hôpital Européen Georges Pompidou, 20-40 rue Leblanc, 75015 Paris, France 


$\begin{array}{ll}\text { Abbreviations } & \\ \text { AUC } & \text { Area under the receiver characteristic curve } \\ \text { BMI } & \text { Body mass index } \\ \text { BNP } & \text { Brain natriuretic protein } \\ \text { COPD } & \text { Chronic obstructive pulmonary disease } \\ \text { COVID-19 } & \text { COVID-19 disease } \\ \text { CT } & \text { Computed tomography } \\ \text { GGO } & \text { Ground glass opacities } \\ \text { HR } & \text { Hazard ratio } \\ \text { hs-troponin I } & \text { High-sensitive troponin I } \\ \text { ICC } & \text { Interclass correlation } \\ \text { ICU } & \text { Intensive care unit } \\ \text { NPV } & \text { Negative predictive value } \\ \text { PAD } & \text { peripheral artery disease } \\ \text { PPV } & \text { Positive predictive value }\end{array}$

SARS-CoV-2 Severe acute respiratory syndrome coronavirus 2

\section{Introduction}

Coronavirus disease 2019 (COVID-19), caused by the novel severe acute respiratory syndrome coronavirus 2 (SARS$\mathrm{CoV}-2$ ), is a new infectious disease that has rapidly become a pandemic. Low-dose computed tomography (CT) on admission has been widely used for screening of suspected COVID19 patients at the early stage of the infection in China [1, 2], and some investigators have also shown in retrospective series that consolidation opacities and/or bilateral patchy shadowing was more frequently found in non-survivor COVID patients as compared to survivors [3, 4], and the extent of lung damage was more pronounced in more severe COVID-19 [5]. Different lung damage spread scores proposed in retrospectives studies to be related to the severity of infection [6-11]. However, there is no study that has prospectively validated these scores in independent and non-selected cohorts of severe hospitalized COVID-19 patients but without SARS by using short-term mortality as the final outcome reference and also tested the independence of these scores with respect to conventional clinical and/or biological severity factors. Furthermore, from the beginning to the peak of the COVID19 outbreak now reached in many countries, there is still no consensus about the way to standardize $\mathrm{CT}$ reading to better stratify the risk of patients to help urgent decision-making and triage of primary importance at hospital admission. When the international Fleischner Society explored the application of CT in patients suspected of COVID-19 at the end of March 2020, the authors of that report [12] pointed out the lack of objective data concerning the estimation of risk based on lung damage observed on CT scan.

Thus, in a prospective cohort of confirmed COVID-19 hospitalized patients during the first 4 weeks of the outbreak in Paris, the purpose of the present study was to define and to evaluate the association between standardized simple visual lung opacity CT scores at admission, which does not require any software, and 30-day mortality and to compare such CT score to other conventional risk factors of COVID-19 also recorded at hospital admission.

\section{Methods}

\section{Patient population}

This was a prospective designed pilot cohort study in a single center and no predefined number of subjects was therefore planned. Thus, all consecutive adult patients ( $\geq 18$ years) hospitalized in our institution between March 13 and April 8, 2020 (Fig. 1), for at least $24 \mathrm{~h}$ with chest $\mathrm{CT}$ at admission due to laboratory-confirmed COVID-19 were eligible (IRB registration \#00011928) and signed informed consents. The study population was COVID-19 patients without SARS at the time of admission by requiring hospitalization with oxygen therapy and standard medical monitoring due to associated comorbidities. Primary (30-day mortality) and secondary outcomes (30-day mortality or transfer to ICU, and recovery as defined in Supplementary files) were monitored until May 8, 2020, allowing a minimum of a 30-day follow-up for all patients.

\section{Chest CT characteristic and visual lung damage CT score}

All low-dose chest CTs were performed on the same multirow system (Somatom Definition Edge, Siemens) with a collimation of $128 \times 0.6 \mathrm{~mm}$ (reconstruction in $1.5 \mathrm{~mm}$ in mediastinal setting and in $1 \mathrm{~mm}$ in lung setting), and a $280-\mathrm{ms}$ gantry rotation time. The chest CT scan was obtained in a supine position from the lung apices to the bases in a single breath-hold at maximum inspiration without ECG gating. Tube voltage was selected $(100-120 \mathrm{kVp})$ by an automated tube voltage selection associated with $40-80 \mathrm{mAs}$, based on body size.

In accordance with the Fleischner Society [13], and as previously described in recent articles $[2,14,15]$ regarding chest CT in COVID-19, in all patients, lung injuries were assessed in each lung lobe for the presence of either GGO (hazy areas of increased attenuation without obscuration of the underlying vasculature) or consolidation (homogeneous opacification with obscuration of the underlying vasculature) or both. Other chest CT characteristics were assessed as follows: presence of $\geq 10$-mm lymphadenopathy, nodules, pleural effusion, and airway abnormalities. The axial distribution was classified as central, peripheral, or mixed when lesions occurred at more than $3 \mathrm{~cm}$, less than $3 \mathrm{~cm}$ from the chest wall, or on either side, respectively. The presence of underlying lung disease such as 


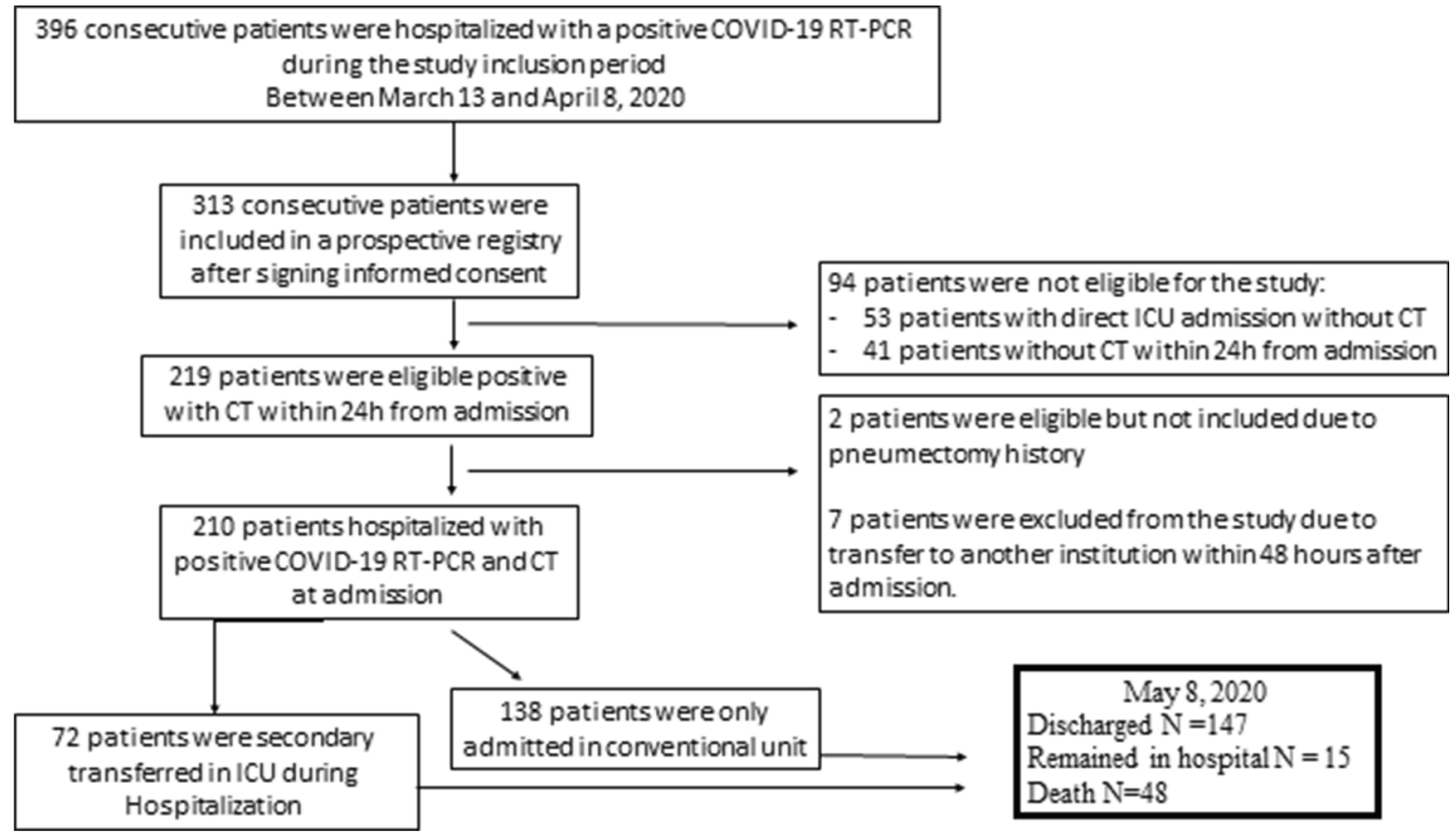

Fig. 1 Patient recruitment flowchart

emphysema or fibrosis as well as linear opacities, opacities with a rounded morphology or with a "crazy-paving" pattern, and halo and reverse halo signs were also noted. Finally, to define visual CT scores of the lung damages observed within each lung lobe, CT images of all patients were assessed by pairs of radiologists completed by a third independent reader in case of discrepancy. Each of the 5 lung lobes was visually assessed to determine the presence of GGO or consolidation and degree of extension of either GGO areas alone or GGO areas plus condensation opacity areas. Thus, each lung lobe extent was classified as having $0,1,2,3$, or 4 score when none, minimal (1-25\%), mild (26-50\%), moderate (51$75 \%$ ), or severe ( $>75 \%$ ) were visually estimated, respectively. The number of lung lobes involved per patient by GGO or consolidation opacities was also estimated as a first visual CT score to assess the extent of lung damage. According to the presence of these $\mathrm{CT}$ features, patients were also classified as having either low to moderate lung damage extent when 1 to 4 lobes were affected and as having high damage extent when the 5 lobes were affected by either GGO or consolidation. Finally, by summing the visual scores of the 5 lobes per patient, the total severity score was evaluated over a range from 0 to 20 by using consolidation areas alone or in association with GGO areas. Examples of such visual score of both GGO and consolidation obtained in 4 COVID-19 patients are shown in Fig. 2.

In order to establish a so-called consensual reading, the third independent radiologist, expert in cardiothoracic imaging (A.H., E.M., or E.C. with 35, 32, and 7 years of experience, respectively), proceeded to examine all patients that they had not already estimated at the first reading, when an interpretation discrepancy was found between the two first readers in at least one of the 5 lung lobes per patient. This consensus reading was then used for the final analysis of the relationship between the visual lung CT scores, 30-day mortality, and other outcomes. All radiologists involved in the present research were blinded from all clinical data, biology, and initial chest $\mathrm{CT}$ analysis carried out during patient management, and the imaging data were only transmitted to our institution's clinical investigation center once the entire collection of imaging data had been completed 2 weeks before the end of the follow-up.

\section{Statistical analysis}

Sensitivity, specificity, and area under the receiver characteristic curve (AUC) of the best CT score were calculated to predict 30-day mortality. Patient survival according to the appropriate cut-off value of the best CT score was determined using the log-rank test and displayed using the Kaplan-Meier curves. The rates of all-cause mortality at 30 days were analyzed according to the number of affected lung lobes by either GGO, consolidation, or both, and according to the visual lung damage CT score. The impact of the best cut-off values of lung damage CT score to distinguish survivors from nonsurvivors has been further applied before evaluating all determinants of primary and secondary outcomes by using univariate and multivariate Cox proportional hazards regression models after having eliminated non-significant variables of univariate analysis (Supplementary files). 

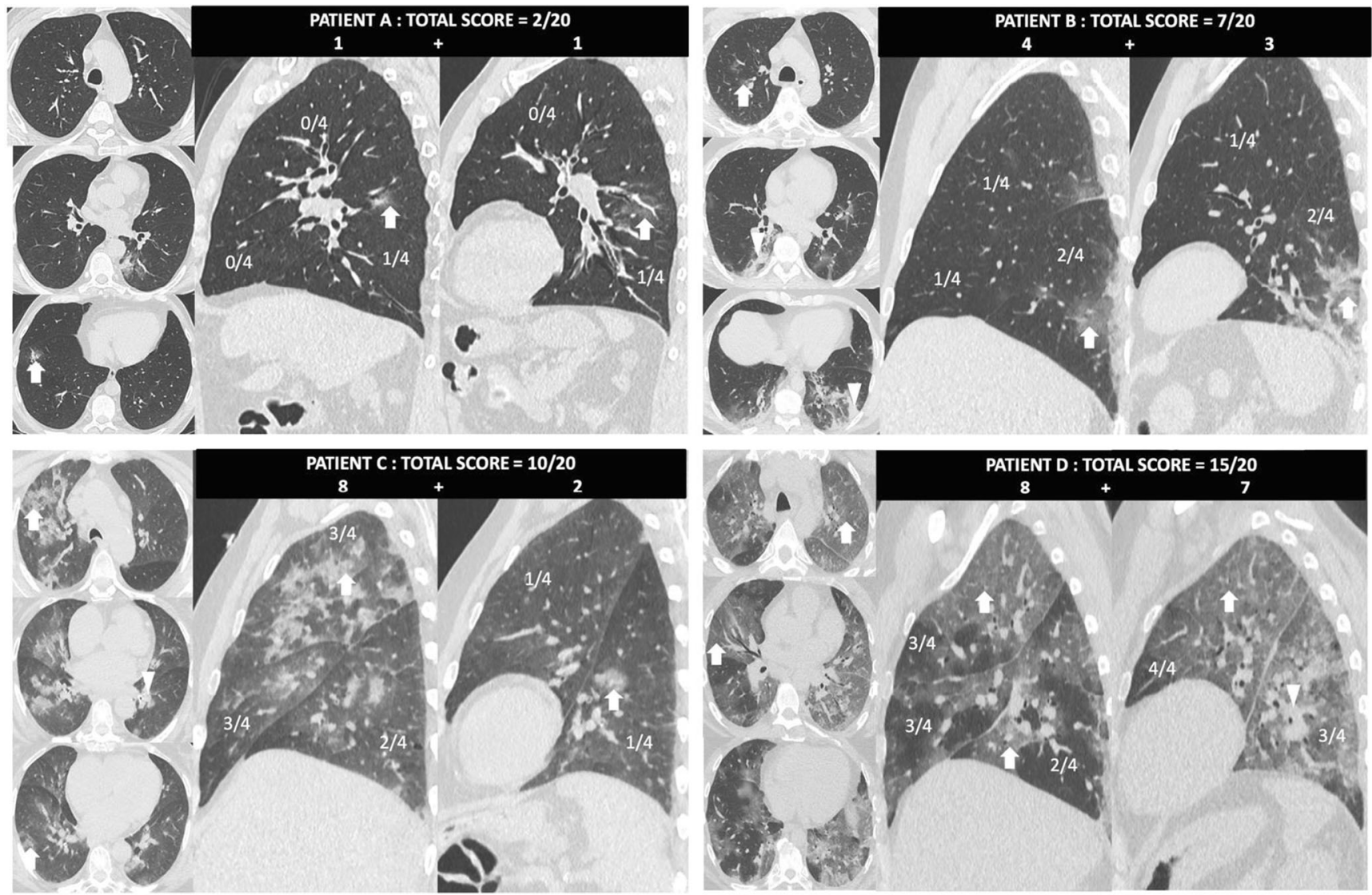

Fig. 2 Three Axial CT images and corresponding right and left sagittal reformatted images in four different patients according to our visual CT severity score. This score is assessed by visually adding GGO (arrows)

and consolidation (arrowheads) extent in each lobe/4 (0: 0\%; 1: 1-24\%; $2: 25-49 \% ; 3: 50-74 \%$; and $4: 75-100 \%)$ and the total score per patient is over 20

\section{Results}

\section{Lung damage at CT and laboratory findings}

\section{Patient characteristics}

As shown in the flow chart in Fig. 1, 210 COVID-19 patients were included (mean age: 66 years \pm 16 ). Baseline clinical characteristics, comorbidities, and prior medications are presented in Table 1 and in Table E1 with more details. Dyspnea in 146 patients $(69.5 \%)$ was the most common symptom. History of coronary artery disease, cerebrovascular disease, COPD, and peripheral artery disease (PAD) was found in 17 (8.1\%), 4 (1.9\%), $15(7.1 \%)$, and $10(4.8 \%)$ patients, respectively. At 30 days, the number of survivors and non-survivors was $162(77 \%)$ and $48(23 \%)$, respectively. In the nonsurvivor group, patients were older, with more COPD or cancer comorbidity but did not present more history of coronary artery disease. With respect to the clinical presentation at admission, compared to survivor patients at 30 days, the more frequent and significant features in non-survivor patients were dyspnea, higher respiratory rate, and lower oxygen saturation.

The main laboratory and CT findings are presented in Table 1 with more details in supplementary Tables E1 and E2. There was a significant increase in creatinine levels, in cardiac biomarkers (i.e., BNP and high-sensitive troponin I levels), and in inflammation biomarkers in non-survivors compared to survivors.

The proportion of patients with bilateral pneumonia was 93.8\% (197 patients), and with 5 lung lobes involved with GGO, consolidation, or by either of them which were 136 (64.8\%), 49 (23.3\%), and $162(77.1 \%)$, respectively. The number of patients affected by consolidation alone in 5 lung lobes was not significantly different between survivors and non-survivors. Furthermore, a significantly higher number of patients were found in the non-survivor group when either GGO areas or GGO associated to consolidation was taken into account in the estimation of lung damage (Table E2). Additionally, the global severity score was significantly higher in non-survivors compared to that in survivors when the score was estimated by taking into account both GGO and 
Table 1 Baseline clinical, biological, and radiological characteristics of the population $(n=210)$

\begin{tabular}{|c|c|c|c|c|}
\hline & Overall $(n=210)$ & Survivors $(n=162)$ & Non-survivors $(n=48)$ & $p$ value \\
\hline Age, y & $66 \pm 16$ & $63 \pm 16$ & $74 \pm 15$ & $<0.001$ \\
\hline Male & $146(69.5)$ & $114(70.4)$ & $32(66.7)$ & 0.624 \\
\hline BMI, $\mathrm{kg} / \mathrm{m}^{2}$ & $25.6 \pm 5.1$ & $26.6 \pm 4.7$ & $26.6 \pm 6.4$ & 0.973 \\
\hline \multicolumn{5}{|l|}{ Risk factors } \\
\hline Hypertension & $96(45.7)$ & $70(43.2)$ & $26(54.2)$ & 0.181 \\
\hline Diabetes mellitus & $47(22.4)$ & $37(22.8)$ & $10(20.8)$ & 0.770 \\
\hline \multicolumn{5}{|l|}{ Medical history } \\
\hline History of coronary artery disease & $17(8.1)$ & $10(6.2)$ & $7(14.6)$ & 0.115 \\
\hline COPD & $15(7.1)$ & $8(4.9)$ & $7(14.6)$ & 0.050 \\
\hline Cancer & $41(19.5)$ & $23(14.2)$ & $18(37.5)$ & $<0.001$ \\
\hline \multicolumn{5}{|l|}{ Prior medications } \\
\hline Aspirin & $36(17.1)$ & $28(17.2)$ & $8(16.7)$ & 0.921 \\
\hline Oral anticoagulant & $24(11.4)$ & $14(8.6)$ & $10(20.8)$ & 0.020 \\
\hline Statins & $45(21.4)$ & $37(22.8)$ & $8(16.7)$ & 0.360 \\
\hline Beta blockers & $44(21)$ & $31(19.1)$ & $13(27.1)$ & 0.235 \\
\hline $\mathrm{ACE}$ inhibitors or $\mathrm{ARB}$ & $60(28.6)$ & $44(27.2)$ & $16(33.3)$ & 0.406 \\
\hline Diuretics & $37(17.6)$ & $29(17.9)$ & $8(16.7)$ & 0.844 \\
\hline \multicolumn{5}{|l|}{ Clinical presentation } \\
\hline Respiration rate per min & $22.3 \pm 6.9$ & $24.4 \pm 6.1$ & $25.3 \pm 8.2$ & 0.003 \\
\hline Oxygen saturation, $\%$ & $92.2 \pm 7.3$ & $93.4 \pm 4.4$ & $87.9 \pm 12.1$ & 0.003 \\
\hline Dyspnea & $146(69.5)$ & $106(65.4)$ & $40(83.3)$ & 0.018 \\
\hline \multicolumn{5}{|l|}{ Laboratory values, median $[\mathrm{IQR}]$ at base line } \\
\hline Creatinine, $\mu \mathrm{g} / \mathrm{dL}$ & $81.5[66-110]$ & $79.564 .8-102.5]$ & $101.5[72.5-128.8]$ & 0.006 \\
\hline D-dimer, $\mu \mathrm{g} / \mathrm{mL}$ & $1116.5[687.5-1805.8]$ & $1004[631.5-1657.3]$ & $1455[834-2537.3]$ & 0.004 \\
\hline C-reactive protein, $\mathrm{mg} / \mathrm{dL}$ & $98[47.8-149.2]$ & $91.1[39.8-133.5]$ & $123[67-193.5]$ & 0.009 \\
\hline Lymphocytes, $\times 10^{6} / \mathrm{L}$ & $910[610-126]$ & $970[685-1265]$ & $680[440-1243]$ & 0.007 \\
\hline Hemoglobin, g/dL & $13.7[12.5-14.7]$ & $13.9[12.8-14.9]$ & $12.8[11.3-14]$ & $<0.001$ \\
\hline High-sensitivity troponin I, ng/L & $12.2[6-27]$ & $9.6[5.2-19.9]$ & $23.7[15.9-69.2]$ & $<0.001$ \\
\hline Brain natriuretic peptide, $\mathrm{ng} / \mathrm{mL}$ & $48.5[19.3-164]$ & $36[17.5-119]$ & $106[46-316]$ & $<0.001$ \\
\hline \multicolumn{5}{|l|}{ CT imaging findings } \\
\hline Days from illness onset to $\mathrm{CT}$, median [IQR] & $7[4-9]$ & $7[5-9]$ & $6[3-8.8]$ & 0.176 \\
\hline Presence of pleural effusions & $17(8.1)$ & $8(4.9)$ & $9(18.8)$ & 0.005 \\
\hline \multicolumn{5}{|c|}{ Number of patients according to the number of lobes involved with GGO or consolidation } \\
\hline$<5$ & $48(22.8)$ & $42(25.9)$ & $6(12.5)$ & \\
\hline 5 & $162(77.1)$ & $120(74.1)$ & $42(87.5)$ & 0.052 \\
\hline Total CT severity score/20 by adding GGO and consolidation & $7 \pm 3.7$ & $6.3 \pm 3.1$ & $9.5 \pm 4.5$ & $<0.001$ \\
\hline Total CT severity score/20 with consolidation alone & $3.7 \pm 2.6$ & $3.5 \pm 2.3$ & $4.3 \pm 3.8$ & 0.194 \\
\hline
\end{tabular}

Footnotes: Values are expressed as mean ( \pm standard deviation) or median [interquartile range] or number (percentage)

Abbreviations: $C O P D$, chronic obstructive pulmonary disease; $S D$, standard deviation; $B M I$, body mass index; $A C E$, angiotensin-converting enzyme; $A R B$, angiotensin receptor blockers; $I Q R$, interquartile range; $G G O$, ground glass opacity. *Mixed distribution referred to peripheral and central lung lesions

consolidation and not significantly different when only consolidation was taken into account. Pulmonary embolism was detected in 5 patients, but contrast medium was injected during CT in only 43 patients $(20.5 \%)$, with no difference of prevalence between the 2 groups. The presence of pleural effusion and of peripheral distribution of lesions was more frequent and the total CT severity score over 20 was higher in non-survivors compared to that in survivors $(9.5 \pm 4.5 \mathrm{vs}$ $6.3 \pm 3.1 ; p<0.001)$. As shown in Figure E1, the AUC of the lung CT score with both GGO and consolidation was statistically significant in the classification of survivors and nonsurvivors within 30 days (AUC 0.72 ; 95\% CI $0.628-0.807$, 
Table 2 Clinical and biological characteristics and clinical outcomes of patients according to the lung severity CT score class $<7$ or $\geq 7$ which take into account percentage of both GGO and consolidation in each lung lobe

\begin{tabular}{|c|c|c|c|c|}
\hline & Overall $(n=210)$ & CT score $<7(n=115)$ & CT score $\geq 7(n=95)$ & $p$ value \\
\hline Age, y & $66 \pm 16$ & $65.7 \pm 16.3$ & $65.4 \pm 16.2$ & 0.885 \\
\hline Male & $146(69.5)$ & $79(68.7)$ & $67(70.5)$ & 0.774 \\
\hline BMI, $\mathrm{kg} / \mathrm{m}^{2}$ & $25.6 \pm 5.1$ & $26.4 \pm 4.4$ & $26.8 \pm 5.8$ & 0.635 \\
\hline \multicolumn{5}{|l|}{ Clinical presentation at baseline } \\
\hline Respiratory rate, breaths/min & $22.3 \pm 6.9$ & $20.3 \pm 5.5$ & $24.7 \pm 7.5$ & $<0.001$ \\
\hline Oxygen saturation, $\%$ & $92.2 \pm 7.3$ & $94.4 \pm 3.7$ & $89.4 \pm 9.4$ & $<0.001$ \\
\hline Temperature, ${ }^{\circ} \mathrm{C}$ & $38 \pm 1$ & $37.9 \pm 0.9$ & $37.9 \pm 1.1$ & 0.747 \\
\hline \multicolumn{5}{|l|}{ Prior medications } \\
\hline Aspirin & $36(17.1)$ & $25(21.7)$ & $11(11.6)$ & 0.052 \\
\hline Oral anticoagulant & $24(11.4)$ & $12(10.4)$ & $12(12.6)$ & 0.618 \\
\hline Statins & $45(21.4)$ & $34(29.6)$ & $11(11.6)$ & 0.002 \\
\hline ACE inhibitors or ARB & $60(28.6)$ & $37(32.2)$ & $23(24.2)$ & 0.204 \\
\hline \multicolumn{5}{|l|}{ Laboratory values, median [IQR] at baseline } \\
\hline Creatinine, $\mu \mathrm{g} / \mathrm{dL}$ & $81.5[66-110]$ & $82[65-117]$ & $81[66-107]$ & 0.677 \\
\hline D-dimer, $\mu \mathrm{g} / \mathrm{mL}$ & $1116.5[687.5-1805.8]$ & $861[557.5-1165.5]$ & $1347[805.5-1935]$ & 0.001 \\
\hline C-reactive protein, $\mathrm{mg} / \mathrm{dL}$ & $98[47.8-149.2]$ & $65[28.2-112]$ & $128.9[77.5-196.1]$ & $<0.001$ \\
\hline Platelet count, $\times 10^{9} / \mathrm{L}$ & $189[143.5-250]$ & $172.5[135.8-235.8]$ & 207 [156-259] & 0.023 \\
\hline Neutrophils, $\times 10^{9} / \mathrm{L}$ & $4.7[3.2-4.7]$ & $4.13[2.8-6.1]$ & $5.6[3.8-8]$ & $<0.001$ \\
\hline Lymphocytes, $\times 10^{6} / \mathrm{L}$ & $910[610-126]$ & $1[0.8-1.3]$ & $0.7[0.5-1.2]$ & $<0.001$ \\
\hline Hemoglobin, g/dL & $13.7[12.5-14.7]$ & $13.7[12.5-14.7]$ & $13.7[12.3-14.6]$ & 0.813 \\
\hline High-sensitivity troponin I, ng/L & $12.2[6-27]$ & $9.7[5.1-22.3]$ & $17[8.1-30.9]$ & 0.002 \\
\hline Brain natriuretic peptide, $\mathrm{ng} / \mathrm{mL}$ & $48.5[19.3-164]$ & $47[17-161]$ & $50[24-189]$ & 0.25 \\
\hline \multicolumn{5}{|l|}{ Patient triage } \\
\hline $\begin{array}{l}\text { Time from symptom onset to admission } \\
\text { days, median [IQR] }\end{array}$ & $7[4-9]$ & $6[4-8]$ & $8[6-10]$ & 0.005 \\
\hline Admission in ICU & $72(34.3)$ & $24(20.9)$ & $48(50.5)$ & $<0.001$ \\
\hline \multicolumn{5}{|l|}{ Treatment } \\
\hline \multicolumn{5}{|l|}{ Anticoagulant therapy } \\
\hline Preventive & $130(61.9)$ & $77(67)$ & $53(55.8)$ & 0.140 \\
\hline Curative & $74(35.2)$ & $32(27.8)$ & $42(44.2)$ & 0.011 \\
\hline Antiviral treatment & $31(14.8)$ & $14(12.2)$ & $17(17.9)$ & 0.207 \\
\hline \multicolumn{5}{|l|}{ Clinical outcomes } \\
\hline Acute heart failure & $18(8.6)$ & $7(6.1)$ & $11(11.6)$ & 0.157 \\
\hline Arrhythmia & $18(8.6)$ & $10(8.7)$ & $8(8.4)$ & 0.944 \\
\hline Acute renal insufficiency $(\mathrm{GFR}<30 \mathrm{~mL} / \mathrm{min})$ & $41(19.5)$ & $16(12.9)$ & $25(26.3)$ & 0.024 \\
\hline Discharged & $147(70)$ & $98(85.2)$ & $49(51.6)$ & $<0.001$ \\
\hline Remained in hospital & $15(7.1)$ & $5(4.3)$ & $10(10.5)$ & 0.084 \\
\hline Median length of hospital stay, days (ALL) & $9[5-16]$ & 9 [5-13] & $9[5-22]$ & 0.200 \\
\hline Median length of hospital stay, days (survivors) $n=162$ & $9[5-16]$ & 9 [4-13] & $11[5-26]$ & 0.022 \\
\hline Death at 30-day follow-up & $48(22.9)$ & $12(10.4)$ & $36(37.9)$ & $<0.001$ \\
\hline
\end{tabular}

Footnotes: Values are expressed as mean ( \pm standard deviation) or median [interquartile range] or number (percentage). Abbreviations: $A C E$, angiotensin-converting enzyme; $A R B$, angiotensin receptor blockers; $S D$, standard deviation; $G F R$, glomerular filtration rate; $B M I$, body mass index; $I Q R$, interquartile range; $I C U$, intensive care unit

$p<0.001)$. The best cut-off value of this CT score was 7 according to the Youden index leading to 75\% (92-68) sensitivity, 64\% (72-55) specificity, and 38\% (49-27) PPV and 90\% (94-85) NPV.
Moreover, the inter-observer agreement for the CT score was excellent when both consolidation and GGO without differentiation were taken into account in the assessment of lung opacity extension (ICC $=0.856$; CI 
95\%: $0.815-0.888$ ) and good when only consolidation was taken into account in the score (ICC $=0.659$; CI 95\%:0.562-0.738).

\section{Clinical presentation, treatment, and outcomes according to lung damage CT score (Table 2)}

The median time from symptom onset to the CT scan was 7.0 (4-9) days and similar in non-survivors compared to survivors; however, this time was $48 \mathrm{~h}$ longer in the patient group with $\mathrm{CT}$ score $\geq 7$ compared to the other $(p=0.005)$. By comparing patients with $\mathrm{CT}$ score $\geq 7$ and those with CT score $<7$, age, sex ratio, BMI, and temperature were the same when respiratory rate was higher and oxygen saturation lower $(p<0.001$ for both). D-dimer, hs-troponin I, and BNP levels as well as inflammation biomarkers and platelet and neutrophil counts were significantly higher when CT score $\geq 7$.

During hospital management, 72 patients $(34.3 \%)$ were transferred to ICU, with significant difference between patients with CT score $\geq 7$ and those with CT score $<7$ (50.5 vs $20.9 \% ; p<0.001$ ).

The duration of hospitalization was 9 days in both groups when all patients in the cohort were considered, but when only survivors were considered, this duration was $9(4-13)$ and 11 $(5-26)$ days $(p=0.022)$ for patients with severity score $<$ and $\geq 7$, respectively.

The only difference in curative anticoagulant therapy was more frequent in case of extensive lung injuries (44.2 vs $27.8 \%, p=0.011)$. Acute renal insufficiency occurred in 41 cases, 25 (26.3\%) in case of CT score $\geq 7$ compared to 16 $(12.0 \%)$ when the score was $<7(p=0.024)$. Finally, during follow-up, a total of 48 patients (22.9\%) died, 147 patients (70\%) were discharged, and $15(6.5 \%)$ remained hospitalized. Discharges decreased (98 [85.2\%] vs 49 [51.6\%]; $p<0.001$ ) when 30-day mortality increased with the magnitude of visual lung CT score (36 [37.9\%] vs 12 [12.4\%]; $p<0.001)$ for patients with $\mathrm{CT}$ score $\geq 7$ versus those with $\mathrm{CT}$ score $<7$. Between the two groups, the significant differences in KaplanMeier event rate curves are illustrated in Fig. 3, for the survival rate $(p<0.001)$ and for the secondary outcome combining mortality and transfer in ICU before 30 days $(p<0.001)$.

\section{Determinants of the primary (30-day mortality) and secondary (30-day mortality or transfer in ICU) out- comes in univariate and multivariate analysis (Table 3)}

After adjustment with other main determinants of 30-day mortality, there was a significant increasing risk of death with increased lung damage CT score $\geq 7$ (HR, 3.16 (1.50-6.43); $p=0.001)$ (Table 3$)$. Other independent predictors of a

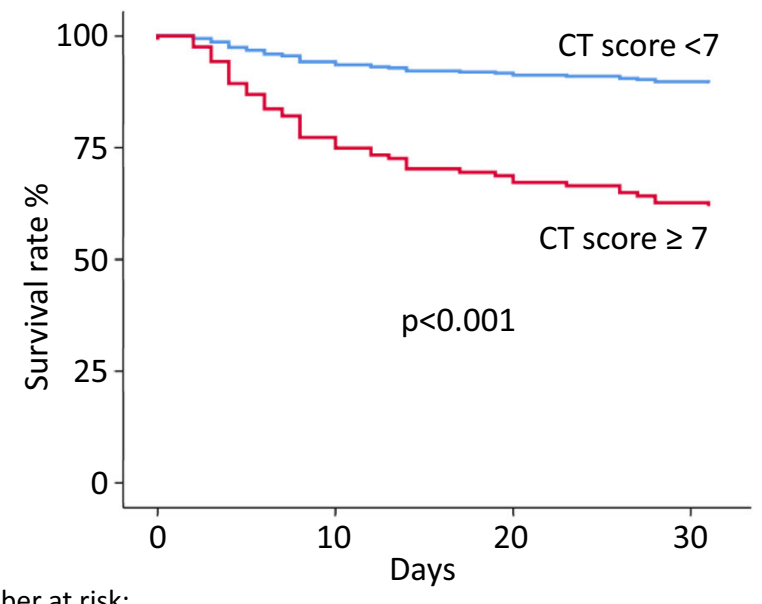

Number at risk:

$\begin{array}{lrrrr}\text { CT score }<7 & 115 & 109 & 105 & 103 \\ \text { CT score } \geq 7 & 95 & 72 & 65 & 59\end{array}$

b

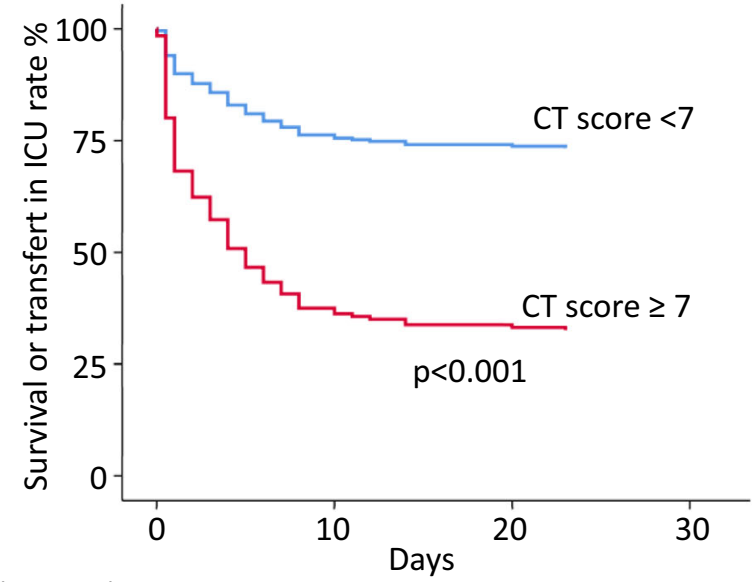

Number at risk:

$\begin{array}{lrrrr}\text { CT score }<7 & 115 & 89 & 86 & 85 \\ \text { CT score } \geq 7 & 95 & 35 & 32 & 31\end{array}$

Fig. 3 30-day survival rate (\%) (primary outcome) in a and either 30-day survival rate or transfer in ICU in $\mathbf{b}$ (secondary outcome) according to magnitude of the visual lung damage CT score

mortality when lung CT score was added in the model were age, respiratory rate (HR, $1.05(1.01-1.11) ; p=0.035)$, BNP (HR, $1.05(1.01-1.11) ; p=0.049)$, and hemoglobin (HR, 0.78 $(0.67-0.92), p=0.002)$ levels. Other significant variables in univariate analysis such as presence of cancer, $\mathrm{C}$-reactive protein, creatinine, and hs-troponin levels at admission were not any more significant in multivariate analysis when such determinants were added in the final model shown in Table 3. When we take into account the secondary outcome by associating mortality or the transfer to the ICU before 30 days (Supplemental Table E3), the CT score obtained a significant overexposure of risk in the multivariate analysis (HR, 2.60 $(1.60-4.21) ; p<0.001)$. Other significant predictors of such 
Table 3 Multivariate and univariate Cox regression analysis on mortality at 30-day models

\begin{tabular}{|c|c|c|c|c|}
\hline & $\begin{array}{l}\text { Univariate analysis hazard ratio } \\
(95 \% \mathrm{CI})\end{array}$ & $p$ value & $\begin{array}{l}\text { Multivariate analysis hazard ratio } \\
(95 \% \mathrm{CI})\end{array}$ & $p$ value \\
\hline Age, y & $1.06(1.02-1.07)$ & $<0.001$ & $1.05(1.02-1.08)$ & $<0.001$ \\
\hline Cancer & $3.13(1.75-5.63)$ & $<0.001$ & $1.88(0.96-3.70)$ & 0.067 \\
\hline Respiratory rate & $1.06(1.03-1.10)$ & 0.001 & $1.06(1.01-1.11)$ & 0.020 \\
\hline Oxygen saturation & $0.96(0.94-0.97)$ & $<0.001$ & $0.98(0.96-1.01)$ & 0.280 \\
\hline Creatinine & $1.00(1.00-1.01)$ & 0.007 & $1.00(1.00-1.01)$ & 0.226 \\
\hline C-reactive protein & $1.00(1.00-1.01)$ & 0.007 & $1.00(1.00-1.01)$ & 0.696 \\
\hline Brain natriuretic peptide & $1.00(1.00-1.00)$ & $<0.001$ & $1.00(1.00-1.00)$ & 0.049 \\
\hline Hemoglobin & $0.74(0.65-0.84)$ & $<0.001$ & $0.80(0.69-0.93)$ & 0.004 \\
\hline CT severity score $<7 / \geq 7$ adding GGO and consolidation & $4.33(2.25-8.32)$ & $<0.001$ & $3.16(1.50-6.43)$ & 0.001 \\
\hline COPD & $2.41(1.08-5.36)$ & 0.032 & & \\
\hline DBP & $0.98(0.96-1.00)$ & 0.015 & & \\
\hline High-sensitivity Troponin I & $1.00(1.00-1.00)$ & 0.034 & & \\
\hline Lymphocytes & $1.03(1.01-1.04)$ & 0.008 & & \\
\hline Heart rate, bpm & $0.98(0.97-1.00)$ & 0.039 & & \\
\hline Oral anticoagulant & $2.34(1.17-4.70)$ & 0.017 & & \\
\hline Coronary artery disease & $1.92(0.86-4.29)$ & 0.110 & & \\
\hline History of heart failure & $1.64(0.40-6.78)$ & 0.492 & & \\
\hline Hypercholesterolemia & $0.93(0.49-1.75)$ & 0.811 & & \\
\hline History of smoking & $0.77(0.39-1.50)$ & 0.437 & & \\
\hline Days from illness onset to $\mathrm{CT}$ & $0.99(0.89-1.05)$ & 0.891 & & \\
\hline D-dimer, $\mu \mathrm{g} / \mathrm{mL}$ & $1.00(1.00-1.00)$ & 0.299 & & \\
\hline Male & $0.84(0.46-1.53)$ & 0.570 & & \\
\hline BMI & $1.00(0.94-1.07)$ & 0.953 & & \\
\hline Statins & $0.70(0.327-1.494)$ & 0.356 & & \\
\hline Aspirin & $0.97(0.45-2.06)$ & 0.926 & & \\
\hline Diabetes mellitus & $0.85(0.42-1.71)$ & 0.652 & & \\
\hline Hypertension & $1.49(0.85-2.63)$ & 0.167 & & \\
\hline
\end{tabular}

Footnotes: Hazard ratio (HR) is calculated with each per unit-increase. Abbreviations: $C I$, confidence interval; $C O P D$, chronic obstructive pulmonary disease; $B M I$, body mass index in $\mathrm{kg} / \mathrm{m}^{2} ; D B P$, diastolic blood pressure

secondary outcome in univariate analysis, such as age, oxygen saturation, creatinine, and C-reactive protein were nonsignificant when lung CT score, were added in the model. Only the respiratory rate was still significant (HR, 1.05 $(1.01-1.11))$.

\section{Discussion}

To the best of our knowledge, this is the first prospective cohort study in non-selected hospitalized patients to report and compare the relationship between visual score of lung damage at $\mathrm{CT}$ at admission, short-term mortality, and recognized predictors of COVID-19 mortality. By using CT at admission of hospitalized patients, we have shown here that a visual global severity score significantly predicts either the 30 day COVID-19 mortality or the composite event criterion associating the 30-day COVID-19 mortality and the transfer in
ICU, when percentages of both GGO and consolidation areas were taken into account in the assessment through the 5 lung lobes and not when only consolidation areas were taken into account. COVID-19 patients with high extent of lung opacities had higher risk of death, independently of age, cardiovascular risk factors including hypertension, diabetes, BMI, smoking, and other cardiovascular or non-cardiovascular morbidities. Notably, the presence of a visual lung CT score $\geq 7$ at admission was also associated with death independently of respiratory rate and oxygen saturation levels which are among the basic risk factors obtained during the initial clinical examination of these patients to evaluate the severity of the disease. Likewise, the severity CT score was also related to 30-day mortality independently of hs-troponin levels, creatinine levels, and other inflammation biomarkers which have recently been shown $[3,15]$ to be significantly related to COVID-19 mortality. Furthermore, our study has also confirmed that in the presence of a greater extent of lung damage, higher general 
inflammation is observed and might be associated to higher impact of the systemic infection/inflammation on the kidney and heart (Table E2). This relation could be explained by the presence of angiotensin-converting enzyme 2 (ACE2) cell entry receptor of SARS-CoV2 express on the epithelial cell of the lung, heart, and kidney [16], and because viral elements in endothelial cells have been found in these organs [17]. In experimental models [18] now confirmed by human necropsy studies, diffuse alveolar damage with edema, hemorrhage, and intra-alveolar fibrin deposition with extensive apoptosis in the later proliferative phase of tissue repair is related to initial viral load and associated with marked cytokine activation in the first week of viral injection [19]. All this underlines the importance of characterizing alveolar lesions and assessing their extension at the initial phase of viral infection. Thanks to a standardization of CT scan readings proposed by investigators at the beginning of the COVID-19 epidemic, a rapid increase in the prevalence and spread of GGO and condensation alveolar opacities have been observed in the first 12 days after the onset of symptoms [2,20]. Compared to retrospective Chinese studies [2], COVID-19 was more severe in our study despite the absence of SARS at inclusion and the prevalence of extensive lesion was much higher since we found $77.1 \%$ of patients with 5 lung lobes affected by either GGO or consolidation versus $27 \%$, and when using the same CT score in our study, the mean lung severity score was $7 \pm 3.7$ instead of $3 \pm$ 3. Other scores have been proposed in the literature with the percentage of alveolar opacities extension strongly weighted in case of consolidation for a given lobe [4]. Beyond the difficulty of visually differentiating between consolidation areas and GGO areas, we found here that weighting by the extension of consolidation should not be very useful since we found that the lung severity score associating consolidation and GGO areas determined a higher risk than the score considering only consolidation areas (Table E2). To our knowledge, our prospective study is the first to find in non-selected patients and without SARS at admission the high association between severity of CT lesions and short-term mortality independently of other commonly accepted risk factors. The mortality at 1 month of the present study (22.8\%) was very close to the one previously reported in the "recovery study" [21] for subjects not requiring assisted ventilation but the usual treatment including simple nasal oxygen therapy without dexamethasone. Furthermore, to evaluate prognosis, the shortterm mortality seems to us as a more objective criterion than a composite endpoint including mortality. Indeed, given the magnitude of the epidemic from one location to another, at near-peak the number of patients transferred to intensive care and conversely sent home were more complex to analyze because of the obvious lack of available space in many institutions, particularly regarding ICUs. However, based on rules defined just before the epidemic and likely to be applied in our institution to the management of hospitalized COVID-19 patients, we have also confirmed here that the proportion of patients discharged was lower and that transfers to the ICUs were greater when the lung severity CT score on admission was $\geq 7$. Furthermore, we have also shown that $\mathrm{CT}$ at admission in patients without SARS can accurately predict the management of patients during hospitalization since the CT score of lung damage better than oxygen saturation and all other biological biomarkers also obtained at admission was related to the composite outcomes associating 30-day mortality or the transfer in ICU $(\mathrm{HR}=3.68 ; p<0.001)$.

\section{Study limitations}

This is a monocentric cohort study on a relatively limited number of hospitalized patients. It would also be of interest to evaluate CT in a larger population of COVID-19 patients presenting different clinical severity, in particular, early and less severe forms, in order to better analyze the possibilities of triage of patients by CT prior to possible hospitalization, notably in those not requiring immediate hospitalization as in our study. Since the objective of the study was to compare CT results to all other recognized risk factors obtained at admission to predict the occurrence of SARS and short-term mortality, COVID-10 patients directly admitted in ICU were not included in the present study. Thus, the additional value of CT in this population remains to be demonstrated. Concerning the subjective visual assessment of lung CT damage, it is highly probable that automated analysis with $[22,23]$ or without [9] the help of artificial intelligence methods to quantify residual pulmonary functional zones based on density of opacity and normal lung areas will be more efficient to determine patient outcomes, as shown in a recent study $[19,20]$, reporting the relationship of these zones with a composite criterion associating mortality and transfer to an intensive care unit. However, we have confirmed here that a standardized simple visual lung damage CT score, which does not require any software, could be easily reproducible between different radiologists.

In conclusion, our results have important clinical implications, by indicating that a simple visual score of lung alveolar opacity detected by the chest $\mathrm{CT}$ performed at hospital admission was found to be an independent predictor of 30-day mortality associated or not with the transfer in ICU as a composite event criterion. Since such validation of lung damage estimation was obtained in a prospective cohort of COVID-19 patients by a score also related to risk and severity of the disease in previous independent studies [6-8], this score should be incorporated into risk stratification algorithms and in structured chest $\mathrm{CT}$ reports requiring a standardized reading by radiologists. 
Supplementary Information The online version contains supplementary material available at https://doi.org/10.1007/s00330-021-07938-2.

Acknowledgements The authors would like to thank the other investigators of the cohort, all the staff of the radiology department and the clinical research unit without whom this work could not have been done, but also all the staff of the many departments who worked particularly hard during this difficult and very special period to maintain a good quality of care in our institution.

Funding This study has received funding from Assistance Publique Hôpitaux de Paris.

\section{Declarations}

Guarantor The scientific guarantor of the study is Prof. Elie Mousseaux.

Conflict of interest The authors of the manuscript declare no relationships with any companies, whose products and services may be related to the subject matter of the article

Statistic and biometry Three of the authors (EM, GS, and EM) have significant statistical expertise and no complex statistical methods were necessary for this paper.

Informed consent Informed consent was obtained from all patients included in the present study.

Ethical approval This prospective study received agreement of the local Institutional Review Bard-Paris Ile de France (IRB registration \#00011928).

\section{Methodology}

- prospective study

- performed at one institution

\section{References}

1. Ai T, Yang Z, Hou H et al (2020) Correlation of chest CT and RTPCR testing in coronavirus disease 2019(COVID-19) in China: a report of 1014 cases. Radiology 296:E32-E40. https://doi.org/10. 1148/radiol.2020200642

2. Bernheim A, Mei X, Huang M et al (2020) Chest CT findings in coronavirus disease-19(COVID-19): relationship to duration of infection. Radiology 295:200463. https://doi.org/10.1148/radiol. 2020200463

3. Zhou F, Yu T, Du R et al (2020) Clinical course and risk factors for mortality of adult inpatients with COVID-19 in Wuhan, China: a retrospective cohort study. Lancet 395:1054-1062

4. Yuan M, Yin W, Tao Z, Tan W, Hu Y (2019) Association of radiologic findings with mortality of patients infected with 2019 novel coronavirus in Wuhan, China. PLoS One 15(3):e0230548. https://doi.org/10.1371/journal.pone.0230548

5. Guan W, Ni Z, Hu Y et al (2020) Clinical characteristics of coronavirus disease 2019 in China. N Engl J Med 382:1708-1720

6. Zhao W, Zhong Z, Xie X, Yu Q, Liu J (2020) Relation between chest $\mathrm{CT}$ findings and clinical conditions of coronavirus disease
(COVID-19) pneumonia: a multicenter study. AJR Am J Roentgenol 214:1072-1077

7. Francone M, Iafrate F (2020) Masci GM, et al Chest CT score in COVID-19 patients: correlation with disease severity and shortterm prognosis. Eur Radiol 30(12):6808-6817

8. Li Y, Yang Z, Ai T, Wu S, Xia L (2020) Association of "initial CT" findings with mortality in older patients with coronavirus disease 2019(COVID-19). Eur Radiol 30:6186-6193

9. Colombi D, Bodini FC, Petrini M et al (2020) Well-aerated lung on admitting chest CT to predict adverse outcome in COVID-19 pneumonia. Radiology 296:E86-E96. https://doi.org/10.1148/radiol. 2020201433

10. Tabatabaei SMH, Rahimi H, Moghaddas F, Rajebi H (2020) Predictive value of CT in the short-term mortality of Coronavirus Disease 2019(COVID-19) pneumonia in nonelderly patients: a case-control study. Eur J Radiol 132:109298. https://doi.org/10. 1016/j.ejrad.2020.109298

11. Yang R, Li X, Liu H et al (2020) Chest CT severity score: an imaging tool for assessing severe COVID-19. Radiology. https:// doi.org/10.1148/ryct.2020200047

12. Rubin GD, Haramati LB, Kanne JP et al (2020) The role of chest imaging in patient management during the COVID-19 pandemic: a multinational consensus statement from the Fleischner Society. Radiology 296(1):172-180

13. Hansell DM, Bankier AA, MacMahon H, McLoud TC, Müller NL, Remy J (2008) Fleischner Society: Glossary of terms for thoracic imaging. Radiology 246:697-722

14. Wang Y, Dong C, Hu Y et al (2020) Temporal changes of CT findings in 90 patients with COVID-19 pneumonia: a longitudinal study. Radiology 296:E55-E64. https://doi.org/10.1148/radiol. 2020200843

15. Wang D, Hu B, Hu C et al (2020) Clinical characteristics of 138 hospitalized patients with 2019 novel coronavirus-infected pneumonia in Wuhan, China. JAMA 323:1061-1069

16. Zhang F, Liang Y (2020) Potential risk of the kidney vulnerable to novel coronavirus 2019 infection. Am J Physiol Renal Physiol 318: F1136-F1137

17. Varga Z, Flammer AJ, Steiger P et al (2020) Endothelial cell infection and endotheliitis in COVID-19. Lancet 395:1417-1418

18. Chan JF-W, Zhang AJ (2020) Yuan S, et al Simulation of the clinical and pathological manifestations of coronavirus disease 2019(COVID-19) in golden Syrian hamster model: implications for disease pathogenesis and transmissibility. Clin Infect Dis 71(9):2428-2446. https://doi.org/10.1093/cid/ciaa325

19. Ackermann M, Verleden SE (2020) Kuehnel M, et al Pulmonary vascular endothelialitis, thrombosis, and angiogenesis in Covid-19. N Engl J Med 383(2): 120-128

20. Pan F, Ye T, Sun P et al (2020) Time course of lung changes at chest CT during recovery from coronavirus disease 2019(COVID19). Radiology $295: 715-721$

21. The RECOVERY collaborative group (2020) Dexamethasone in hospitalized patients with Covid-19. Preliminary Report. N Engl J Med 384(8):693-704. https://doi.org/10.1056/NEJMoa2021436

22. Belfiore MP, Urraro F, Grassi R et al (2020) Artificial intelligence to codify lung CT in Covid-19 patients. Radiol Med 125:500-504

23. Homayounieh F, Babaei R, Ebrahimian S et al (2020) Computed tomography radiomics can predict disease severity and outcome in coronavirus disease 2019 pneumonia. J Comput Assist Tomogr 44(5):640-646. https://doi.org/10.1097/RCT.0000000000001094

Publisher's note Springer Nature remains neutral with regard to jurisdictional claims in published maps and institutional affiliations. 